\title{
ACOLHIMENTO E VÍNCULO EM PACIENTES DEMENCIADOS - ESTRATÉGIAS PARA TRATAMENTO EM FISIOTERAPIA: RELATO DE EXPERIÊNCIA
}

\author{
Natália Dal Forno'; Myrian Bitencourt Neves², Aletheia Peters Bajotto³
}

\section{RESUMO}

Objetivo: Relatar as estratégias utilizadas durante o tratamento de um paciente com diagnóstico de Alzheimer e descrever as barreiras encontradas e as estratégias para alcançar acolhimento e vínculo adequados com pacientes portadores de demência. Método: Atendimento de fisioterapia em dupla realizado na região central do Rio Grande do Sul, em 4 sessões por um período de 7 semanas em um paciente idoso com diagnóstico de Doença de Alzheimer. Resultados: Das barreiras encontradas, se destacam a dificuldade no acolhimento que ocorreu unilateral pelas acadêmicas com a paciente, dificuldade na comunicação e concentração, inexistência do dilálogo, baixa adesão e interesse ao tratamento. Sobre as estratégias elaboradas, está o acolhimento desde a recepção, assuntos relacionados a memórias passadas, exercícios cognitivos, de condicionamento cardiorrespiratório e planejamento motor, atividades e hobbies do dia a dia e o comando verbal adequado. Conclusão: os conceitos discutidos podem facilitar a aderência do paciente ao tratamento proposto.

Palavras-chave: Senescência; Doença de Alzheimer; Técnicas Fisioterápicas.

Eixo Temático: Atenção Integral e Promoção à Saúde (AIPS).

\section{INTRODUÇÃO}

Com o avanço do envelhecimento da população e consequente ampliação da expectativa de vida, um dos grandes desafios para a saúde pública são a alta das taxas de doenças crônicas não transmissíveis, sendo as demências as mais relacionadas ao público idoso (SANTOS; BESSA; XAVIER, 2018). Dos casos diagnosticados, a doença de Alzheimer é o subtipo mais comum, que possui caráter neurodegenerativo, progressivo e de início insidioso. (MATTOS; KÓVACS, 2020; TALMELLI et al., 2013).

\footnotetext{
${ }^{1}$ Natália Dal Forno - Universidade Franciscana natalia.dforno@ufn.edu.br

${ }^{2}$ Myrian Bitencourt Neves - Universidade Franciscana myrian.bitencourt@ufn.edu.br

${ }^{3}$ Aletheia Peters Bajotto - Universidade Franciscana aletheia@bajotto.com.br
} 
De acordo com a Organização Mundial da Saúde (OMS, 2020), em torno de 50 milhões de indivíduos possuem demência, sendo que a Doença de Alzheimer corresponde de 60 a 70\% dos casos, estes responsáveis pelo comprometimento da memória, orientação, pensamento, linguagem, julgamento, compreensão, cálculo e aprendizagem.

Apesar de tal doença não possuir cura, o Ministério da Saúde (2013) relata que tratamentos medicamentosos foram elaborados com o objetivo de aliviar os sintomas e promover a conservação da cognição, comportamento e prática das atividades de vida diárias, onde os inibidores da acetilcolinesterase como a donepezilla, rivastigmina e galantamina são os mais utilizados como a linha de tratamento principal, de maneira que as manifestações clínicas da doença sejam modificadas.

A fisioterapia inicia-se com a avaliação, para que o protocolo de tratamento seja baseado nas características clínicas que o paciente apresenta. A partir disso, o tratamento possui os objetivos de estimular ao máximo a independência funcional, reduzir o avanço das perdas motoras e prevenir deformidades e encurtamentos musculares, através da prática de atividades físicas como a caminhada que proporciona a melhora na qualidade da marcha, aumento de força muscular e melhora do equilíbrio diminuindo o risco de quedas, além da dança como forma de proporcionar a melhora da autonomia e socialização. É importante que cada paciente tenha um plano de tratamento específico e que ele seja reavaliado com frequência de acordo com as capacidades e limitações de cada paciente (MELO; DRIUSSO, 2008).

A OMS (2020) ainda relata sobre os estigmas e barreiras devido à falta de compreensão e consciência sobre as demências, que dificulta 0 acesso a diagnóstico e tratamentos adequados. Com base nisso, é necessário que se tenha uma visão além daquilo que é aparente, ou seja, que haja entre as pessoas o diálogo e a aceitação das diferenças, de maneira que se tenha o respeito das crenças, valores e práticas de cada pessoa, além do acolhimento necessário com o paciente demenciado, pois estes indivíduos também fazem parte da sociedade (BARBOSA et al., 2020). Neste sentido, o objetivo deste artigo é relatar as 
estratégias utilizadas durante o tratamento de um paciente com diagnóstico de Alzheimer e descrever as barreiras encontradas e as estratégias adotadas para alcançar acolhimento e vínculo adequados com pacientes portadores de demência.

\section{RELATO DE EXPERIÊNCIA}

\subsection{ACOLHIMENTO E VÍNCULO}

Durante prática que envolve atendimento de paciente idoso de um curso de Fisioterapia da região central do Rio Grande do Sul, uma dupla de alunos realizou atendimentos com um paciente com diagnóstico de Doença de Alzheimer. Foram realizados quatro encontros, durante sete semanas. A partir daí, durante discussões com os professores envolvidos na supervisão desta prática fisioterapêutica, levantou-se a questão da necessidade de criar estratégias para a aplicação de conceitos de acolhimento e vínculo. Sabe-se que no processo de reabilitação, é de extrema importância que haja uma interação entre paciente e terapeuta. No âmbito da fisioterapia, esse vínculo é construído a partir dos componentes cognitivos e afetivos, mas que muitas vezes eles podem ser afetados pelas expectativas, preocupações, aspectos sociais, culturais e emocionais entre ambos os indivíduos (CANTO; SIMÕES, 2009).

Diante disso, para que ocorra sucesso na reabilitação, é necessário que se construa entre o paciente e o profissional fisioterapeuta uma boa relação baseada na escuta, carinho e respeito (SUBTIL et al., 2011).

Segundo o Ministério da Saúde (2010) o acolhimento caracteriza-se como um "processo constitutivo das práticas de produção e promoção de saúde que implica responsabilização do trabalhador/equipe pelo usuário, desde a sua chegada até a sua saída, ouvindo sua queixa, considerando suas preocupações e angústias, fazendo uso de uma escuta qualificada que possibilite analisar a demanda e, colocando os limites necessários, garantir atenção integral, resolutiva e responsável por meio do acionamento/articulação das redes internas dos serviços e redes externas, com outros serviços de saúde, para continuidade da assistência quando necessário". Já o vínculo é descrito como um "processo que ata ou liga, gerando 
uma ligação afetiva e ética entre ambos, numa convivência de ajuda e respeito mútuos".

Fornecendo a devida atenção à importância do acolhimento e vínculo para o sucesso da reabilitação, o acolhimento é caracterizado como a capacidade de analisar a real necessidade do indivíduo em relação a sua saúde, visto que esse é um processo realizado a partir de uma qualificada escuta com o intuito de garantir adequado acesso as práticas em saúde, através da confiança, vínculo e compromisso entre usuários e profissionais (POLÍTICA NACIONAL DE HUMANIZAÇÃO, 2013). Vale ressaltar que o vínculo é importante na continuidade do tratamento, devido às relações de respeito, escuta e diálogo obtidas durante esse processo, onde a pessoa acometida sente-se mais segura e aceita por pessoas que estão zelando pelo seu bem estar (BRUNELLO et al., 2010).

\subsection{RESULTADOS: BARREIRAS E AS ESTRATÉGIAS ADOTADAS PARA ALCANÇAR O ACOLHIMENTO E VÍNCULO COM PACIENTES DEMENCIADOS:}

\subsubsection{Barreiras:}

Enquanto alunas do $8^{\circ}$ semestre do curso de fisioterapia de uma universidade na região central do $\mathrm{RS}$, percebemos algumas barreiras durante o processo de acolhimento e vínculo com um paciente com demência, atendido em uma clínica de fisioterapia. Podem ser citadas:

- Relação de acolhimento da paciente ao chegar à sessão dava-se unilateralmente por parte das acadêmicas.

- Dificuldade em efetivar a comunicação com o paciente, visto que o mesmo não se expressava verbalmente durante a sessão.

- Relação de vínculo entre paciente e terapeuta comprometido pela inexistência do diálogo e escuta qualificada.

- Reduzida adesão do paciente em relação à atividade proposta, mesmo com estímulo verbal apropriado durante a execução da tarefa.

- Limitações motoras para completar as atividades, depois das mesmas terem sido iniciadas. 
- Dificuldade para manter a concentração do paciente que se dispersava facilmente.

- Falta de interesse do paciente na realização das atividades.

\subsubsection{Estratégias:}

Abaixo apresentamos as estratégias abordadas pelas acadêmicas para superar as barreiras no atendimento de um paciente demenciado, com foco em acolhimento e vínculo:

- Manter a rotina do acolhimento desde a recepção da paciente na sala de espera, apresentação das acadêmicas e orientar todas as atividades que serão realizadas.

- Resgatar assuntos que remetem a memórias de longa duração do paciente, como forma de buscar um vínculo passado com sua rotina.

- Realização de atividades que envolvam a cognição e planejamento motor com o intuito de aprimorar esses aspectos e obter melhoras nas práticas realizadas na clínica e ambiente domiciliar.

- Proporcionar práticas relacionadas ao seu dia a dia, para que o paciente sinta-se acolhido pelas terapeutas, além de se sentir com mais vinculado no contexto em que está inserido no seu cotidiano.

- Realização de tarefas que se relacionem com profissões e hobbies praticados no passado, resgatando suas memórias.

- Organização de materiais com diferentes objetos e cores, para estímulo da concentração, iniciativa e planejamento motor.

- Exercícios que promovam a melhora do condicionamento cardiorrespiratório, como maneira de estimular o paciente a se manter ativo.

- Comando verbal conciso e claro para que o paciente estivesse apto a entender a proposta de cada atividade.

\section{DISCUSSÃO E CONCLUSÃO:}

O objetivo deste relato de experiência é apresentar as barreiras encontradas e as estratégias adotadas para alcançar adequadamente o conceito de acolhimento 
e vínculo com pacientes portadores de demência durante atendimento fisioterapêutico. Acreditamos que as barreiras apresentadas interfiram na relação de acolhimento e vínculo com o paciente demenciado, pois como já citado anteriormente, é importante que ocorra a boa relação e interação entre paciente e terapeuta, como forma de se obter ganho durante o processo de tratamento. Esses ganhos vão além dos resultados.

Com base na relação unilateral por parte das alunas devido à dificuldade das mesmas em realizarem efetiva comunicação e escuta qualificada com o paciente demenciado, no estudo de Alexandre et al. (2019) citam que o acolhimento não é considerado apenas como uma ferramenta de porta de entrada para o serviço que o paciente procura, pois o mesmo chega com uma história que merece a devida atenção por parte do profissional da saúde, de maneira que as suas necessidades sejam solucionadas através do processo de construção do acolhimento. Através disso, umas das estratégias utilizadas durante os atendimentos foi manter a rotina do acolher o paciente desde a recepção, apresentação por parte das acadêmicas e explicação das atividades.

Como forma de estimular a comunicação do paciente demenciado, as tarefas que envolvem pistas visuais integram processos cognitivos em relação aos fatos e acontecimentos, visto que a comunicação não é um processo que deve ser separado e isolado de fatores não linguísticos (LIMA et al., 2014). Os autores ainda citam que a doença de Alzheimer pode comprometer a relação entre os sistemas cognitivos, visuais e linguísticos no processo de organização visual e perceptiva. Concordando com os autores, as alunas optaram por realizar atividades cognitivas como organização de materiais com cores e objetos diferentes e tarefas de planejamento motor com o intuito de proporcionar maior concentração, planejamento do movimento e iniciativa para a tarefa, devido à dificuldade que o paciente apresentava nesses quesitos.

Assim como já citado anteriormente, é importante que ocorra a adequada comunicação entre paciente e terapeuta. Durante o atendimento, o comando verbal conciso e claro esteve presente durante todos os atendimentos, de maneira direta e com repetições para que o paciente entendesse o comando ofertado. Galdino; 


\section{QUFN}

Varise (2010) relatam que uma das estratégias do sistema nervoso captar informações e interferir na memória e planejamento motor é através do comando verbal, que associado a componentes verbais e não verbais, intensificam a resposta motora.

Sabe-se que o exercício deve ser uma prática presente no dia a dia dos indivíduos como forma de promover ganhos a saúde. No estudo de Merege et al. (2014) relatam que pessoas com déficits cognitivos por doenças neurodegenerativas podem ter os sintomas reduzidos através da prática regular de exercícios físicos. $A$ partir disso, foram realizados exercícios que possuíssem o objetivo de mantê-lo ativo e proporcionar ganhos ao sistema cardiorrespiratório, através da prática de circuitos que envolvessem diferentes obstáculos e superfícies, com o intuito de promover ganhos positivos no seu bem estar e qualidade de vida.

Concluímos que as principais barreiras que podem atrapalhar a construção do processo de acolhimento e vínculo são a dificuldade de comunicação, sendo realizada apenas pelas alunas e sem feedback do paciente, sem a existência de diálogo, dificuldade em completar as atividades propostas pela falta de concentração, distrações durante a realização e falta de interesse em realizá-las.

As estratégias utilizadas pelas alunas para superar e/ou amenizar essas barreiras foram: resgatar memória de longa duração através de atividades lúdicas relacionadas ao seu cotidiano e lembranças passadas; uso de ferramentas que chamassem atenção para estimular a concentração na realização da tarefa, envolvendo a cognição para planejamento motor, através de formas e cores distintas.

Consideramos importante aplicar esses conceitos discutidos neste relato porque facilita a aderência do paciente demenciado às atividades propostas já que estas remetem sobre seu passado e sua rotina. 


\section{REFERÊNCIAS BIBLIOGRÁFICAS:}

ALEXANDRE, Vinícius et al. O Acolhimento como Postura na Percepção de Psicólogos Hospitalares. Psicologia: Ciência e Profissão, v. 39, 2019.

BARBOSA, Maria Emilia Marcondes et al. Interdisciplinarity of care to the elderly with Alzheimer's disease: reflection to the light of the theories of Leininger and Heller. Escola Anna Nery, v. 24, n. 1, 2020.

BRASIL. Ministério da Saúde. Protocolo Clínico e Diretrizes Terapêuticas. Doença de Alzheimer. Portaria SAS/MS no 1.298, de 21 de novembro de 2013.

Brasil. Ministério da Saúde. Secretaria de Atenção à Saúde. Núcleo Técnico da Política Nacional de Humanização. HumanizaSUS: Documento base para gestores e trabalhadores do SUS / Ministério da Saúde, Secretaria de Atenção à Saúde, Núcleo Técnico da Política Nacional de Humanização. - 4. ed. 4. reimp. - Brasília : Editora do Ministério da Saúde, 2010.

BRUNELLO, Maria Eugênia Firmino et al. O vínculo na atenção à saúde: revisão sistematizada na literatura, Brasil (1998-2007). Acta paulista de Enfermagem, v. 23, n. 1, p. 131-135, 2010.

CANTO, Clóris Regina Elias de Moraes; SIMÃO, Lívia Mathias. Relação fisioterapeuta-paciente e a integração corpo-mente: um estudo de caso. Psicologia: ciência e profissão, v. 29, n. 2, p. 306-317, 2009.

DA SILVA TALMELLI, Luana Flávia et al. Alzheimer's disease: functional decline and stage of dementia. ActaPaulista de Enfermagem, v. 26, n. 3, p. 219-225, 2013.

DE MELO, Marília Agua; DRIUSSO, Patrícia. Proposta Fisioterapêutica para os cuidados de Portadores da Doença de Alzheimer. Envelhecimento e Saúde, 12(4) 2006.

GALDINO, Luana ACS; VARISE, Eliana Maria. Os efeitos do comando verbal na reabilitação física. Revista Neurociências, v. 18, n. 1, p. 95-102, 2010.

HumanizaSUS - PolíticaNacional de Humanização. Brasília: Ministério da Saúde, 2013.

LIMA, Tatiane Machado et al. Doença de alzheimer: cognição e discurso narrativo com apoio em figuras. Revista CEFAC, v. 16, n. 4, p. 1168-1177, 2014. 
MATTOS, Emanuela Bezerra Torres; KOVÁCS, Maria Julia. Alzheimer's disease: the unique experience of family caregivers. Psicologia USP, v. 31, 2020.

MEREGUE, Carlos Alberto Abujabra. Influência Do Exercício Físico Na Cognição: Uma Atualização Sobre Mecanismos Fisiológicos. RevBras Med Esporte - Vol. 20, No 3 - Mai/Jun, 2014.

SANTOS, Camila de Souza dos; BESSA, ThaíssaAraujo de; XAVIER, André Junqueira. Fatores associados à demência em idosos. Ciência \& Saúde Coletiva, v. 25, p. 603-611, 2018.

SUBTIL, Marina Medici Loureiro et al. O relacionamento interpessoal ea adesão na fisioterapia. Fisioterapia em Movimento, v. 24, n. 4, p. 745-753, 2011.

World Heatlh Organization. (WHO). Dementia. Genebra; 2020. Available from:https://www.who.int/news-room/fact-sheets/detail/dementia. Acesso em 19/05/2021 as 20:04. 